\title{
APLICAÇÃO DE UM MODELO DE FECHAMENTO TENSORIAL PARA A SIMULAÇÃO DE PROBLEMAS DE SUPERFÍCIE LIVRE.
}

\author{
R. V. P REZENDE ${ }^{1}$, A. A. ULSON DE SOUZA ${ }^{1}$ e S. M. A. GUELLI ULSON DE SOUZA ${ }^{1}$ \\ ${ }^{1}$ Universidade Federal de Santa Catarina, Departamento de Engenharia Química e \\ Engenharia de Alimentos \\ E-mail para contato: rezendervp@gmail.com
}

\begin{abstract}
RESUMO - Em escoamentos multifásicos a natureza física e matemática do fenômeno leva a postulação de termos de fechamento para a troca de momentum através da interface, bem como outras propriedades, e há a disposição vários modelos com este intento. Mas, diferentemente dos escoamentos dispersados, problemas de superfície livre carecem de correlações para o coeficiente de arrasto. Neste trabalho, o cálculo da força de densidade interfacial é feita dinamicamente sem o uso de qualquer correlação, modelando-se o tensor e os vetores normais à interface, e aplicando-o a problemas benchmark como quebra de barreira e instabilidade de Rayleigh-Taylor. A comparação dos resultados foi satisfatória, tanto com dados numéricos e experimentais da literatura, quanto com a abordagem padrão também empregada. Estas constatações indicam que o modelo é uma boa alternativa para problemas desta natureza, além de poder ser empregado diretamente para o cálculo do coeficiente de arrasto usando a formulação padrão.
\end{abstract}

\section{INTRODUÇÃO}

O aprimoramento e o desenvolvimento de novos modelos e técnicas de medição experimental em escoamentos multifásicos vêm permitindo nos últimos anos o estudo e análise de problemas multifásicos complexos. Códigos comerciais como o ANSYS CFX, ANSYS FLUENT e COMSOL, por exemplo, a cada ano apresentam novas ferramentas matemáticas e numéricas, o que tanto no meio industrial quanto acadêmico se traduzem em melhores ferramentas de pesquisa e, quando associadas a uma infraestrutura computacional adequada, em maior agilidade na análise de problemas computacionalmente proibitivos até a alguns anos dada à complexidade e tempo necessários em sua análise.

Entretanto, este grande avanço depende de quão robustos e abrangentes são os modelos e métodos numéricos desenvolvidos. E o principal concerne à descrição de como as diversas fases do escoamento interagem através de sua interface. E até mesmo a localização resolução e rastreamento desta interface é um problema complexo. Os modelos de fechamento - as equações constitutivas que descrevem como a informação é transferida de uma fase a outra são diversos e dependentes de regime e morfologia como, por exemplo: escoamentos dispersos, polidispersos, para bolhas, gotas ou partículas sólidas e superfície livre. Esta última classe define-se como duas ou mais fases, separadas por uma interface bem definida e não conexa (não fechada) como a superfície entre um rio e a atmosfera. E quando a interface 
é perturbada a morfologia local é alterada para um padrão disperso, como uma mistura de gotas e bolhas, e então os modelos calibrados para uma situação específica passam a não mais descrever a física do problema com a mesma robustez ou até mesmo falham totalmente.

Alguns trabalhos pioneiros lidaram com esta questão (Deendarlianto, et al., 2012; Hänsch, et al., 2012; Höhne, 2009; Höhne e Vallée, 2008, 2010). Os modelos consideram um "blending" entre modelos de superfície livre e partículas, e cálculo dinâmico do coeficiente de arrasto com base no módulo da tensão sobre a interface. Também abordando esta problemática, Rezende, et al. (2012) propuseram um modelo tensorial para o termo de troca de quantidade de movimento alternado a maneira clássica de tratamento do termo.

O presente trabalho é dar continuidade ao estudo desta abordagem tratando mais dois problemas distintos: instabilidade de Rayleigh-Taylor e Rompimento de Barreira. Problemas clássicos, mas com razões de densidade viscosidade e gradientes mais acentuados do que o abordado no primeiro trabalho de Rezende, et al. (2012).

\section{MODELO MATEMÁTICO}

O modelo empregado é apresentado em Rezende, et al. (2012), e considera o sistema isotérmico sem transferência de massa entre as fase. A turbulência não é levada em conta. Assim tem-se a conservação de massa,

$$
\frac{\partial\left(r_{\alpha}\left\langle\rho_{\alpha}\right\rangle\right)}{\partial t}+\nabla \cdot\left(r_{\alpha}\left\langle\rho_{\alpha}\right\rangle\left\langle\mathbf{u}_{\alpha}\right\rangle\right)=0,
$$

conservação da quantidade de movimento linear

$$
\begin{aligned}
& \frac{\partial}{\partial t}\left(r_{\alpha}\left\langle\rho_{\alpha}\right\rangle\left\langle\mathbf{u}_{\alpha}\right\rangle\right)+\nabla \cdot\left(r_{\alpha}\left\langle\rho_{\alpha}\right\rangle\left\langle\mathbf{u}_{\alpha}\right\rangle \otimes\left\langle\mathbf{u}_{\alpha}\right\rangle\right)=-\nabla\left(r_{\alpha}\langle p\rangle\right)+\ldots \\
& \ldots+\nabla \cdot\left[r_{\alpha} \mu_{\alpha}\left\langle\nabla \mathbf{u}_{\alpha}+\nabla \mathbf{u}_{\alpha}^{T}\right\rangle\right]+r_{\alpha}\left\langle\rho_{\alpha}\right\rangle \mathbf{g}+\mathbf{M}_{\alpha}
\end{aligned}
$$

conservação do volume

$$
\sum_{\alpha=1}^{2} r_{\alpha}=1
$$

e o termo de densidade de força interfacial da Equação (2)

$$
\mathbf{M}_{\alpha \beta}=-C_{T} \cdot\left[r_{\alpha}\left\langle\mu_{\alpha}\right\rangle\left\langle\partial_{i} u_{\alpha j} \hat{\mathbf{e}}_{i} \hat{\mathbf{e}}_{j}\right\rangle+r_{\beta}\left\langle\mu_{\beta}\right\rangle\left\langle\partial_{i} u_{\beta j} \hat{\mathbf{e}}_{i} \hat{\mathbf{e}}_{j}\right\rangle\right] \cdot\left(\partial_{k} r_{\alpha} \hat{\mathbf{e}}_{k}\right) \cdot \delta\left(\mathbf{x}-\mathbf{x}_{i}\right) .
$$

O termo $\delta\left(\mathbf{x}-\mathbf{x}_{i}\right)$ é apenas um artifício numérico que ativa o termo fonte junto à interface. Os detalhes da obtenção destas equações podem ser encontrados em Rezende (2008, 2014) bem como em outros trabalhos (Barbosa Jr, 2002; Drew, D., et al., 1979; Drew, D. A., 1983, 1989, 1992; Drew, D. A. e Passman, 1999).

O modelo foi resolvido numericamente no simulador comercial ANSYS CFX R12.1 


\section{INSTABILIDADE DE RAYLEIGH-TAYLOR}

\subsection{Setup}

A Figura 1 apresenta o setup do problema. Um fluido mais denso repousa sobre um menos denso. Uma perturbação inicial é imposta á interface e por ação da gravidade, os fluidos fluem em direções opostas cisalhando suas interfaces no processo. A viscosidade de ambos os fluidos é a mesma, mas a razão de densidades é cerca de 5,73. Em Rezende, et al. (2012) esta razão era bem sutil, cerca de 0,005. A malha cartesiana empregada é composta de 256 x 64 nós. Este problema é facilmente encontrado na literatura e é quase sempre usado como benchmark na análise de códigos numéricos (Fournier, et al., 2002; He, et al., 1999; Mehravaran e Hannani, 2008). Uma fonte onde sua descrição é bem simples e clara, e os resultados são de fácil comparação é dado por Jahanbakhsh, et al. (2007). Para um tempo físico de 1s o tempo de computação é de cerca de três dias com um passo de tempo de $0,001 \mathrm{~s}$ em uma CPU QUAD Core i7-2600@3.4GHz com 16GB de RAM.

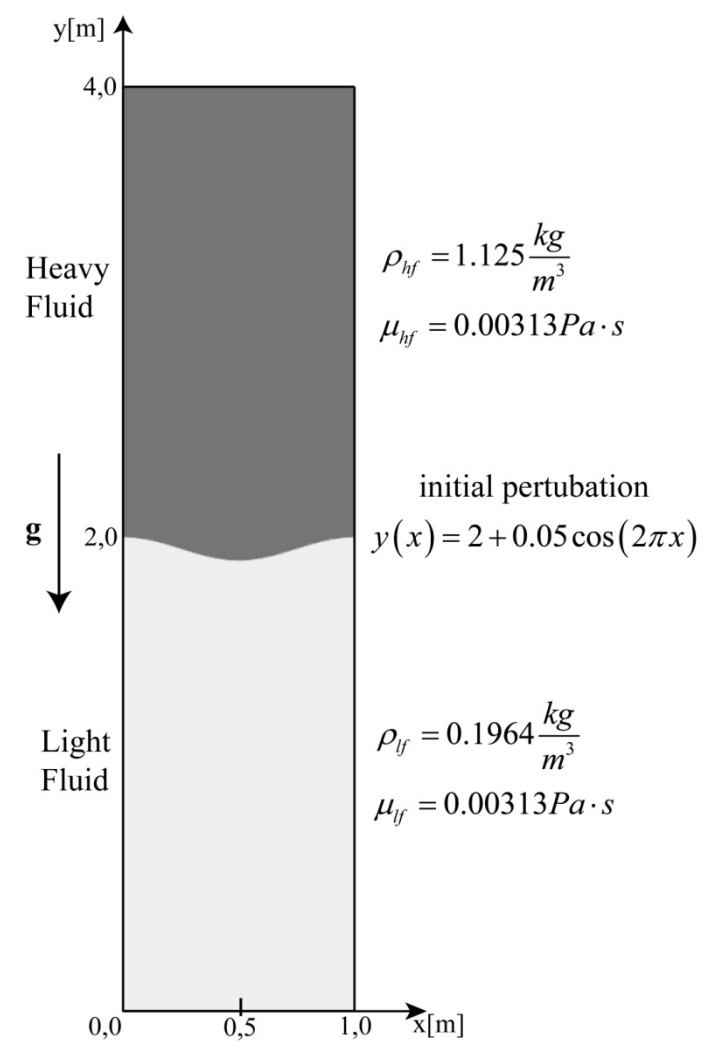

Figura 1 - Configuração e propriedades físicas empregadas na Instabilidade de Rayleigh-Taylor.

\subsection{Resultados e Discussão}

A comparação entre os resultados é apresentada na Figura 2. O comportamento macroscópico apresenta a mesma escala de tempo e os perfis de fração volumétrica são muito similares. Em (A) Jahanbakhsh, et al. (2007) empregam o modelo VOF com o esquema de 
reconstrução de interface proposto em seu trabalho, e localmente, principalmente nas extremidades da frente, há a formação de gotas, e duas estruturas na interface superior não presentes nos resultados de Jahanbakhsh, et al. (2007). A interface neste caso aparece mais complexa do que a geralmente é apresentada na literatura. Este efeito também ocorre quando se aplica o modelo de arrasto clássico com coeficiente de arrasto constante igual a 0,44. Mas, os resultados de forma geral apresentam boa concordância para as grandes estruturas.
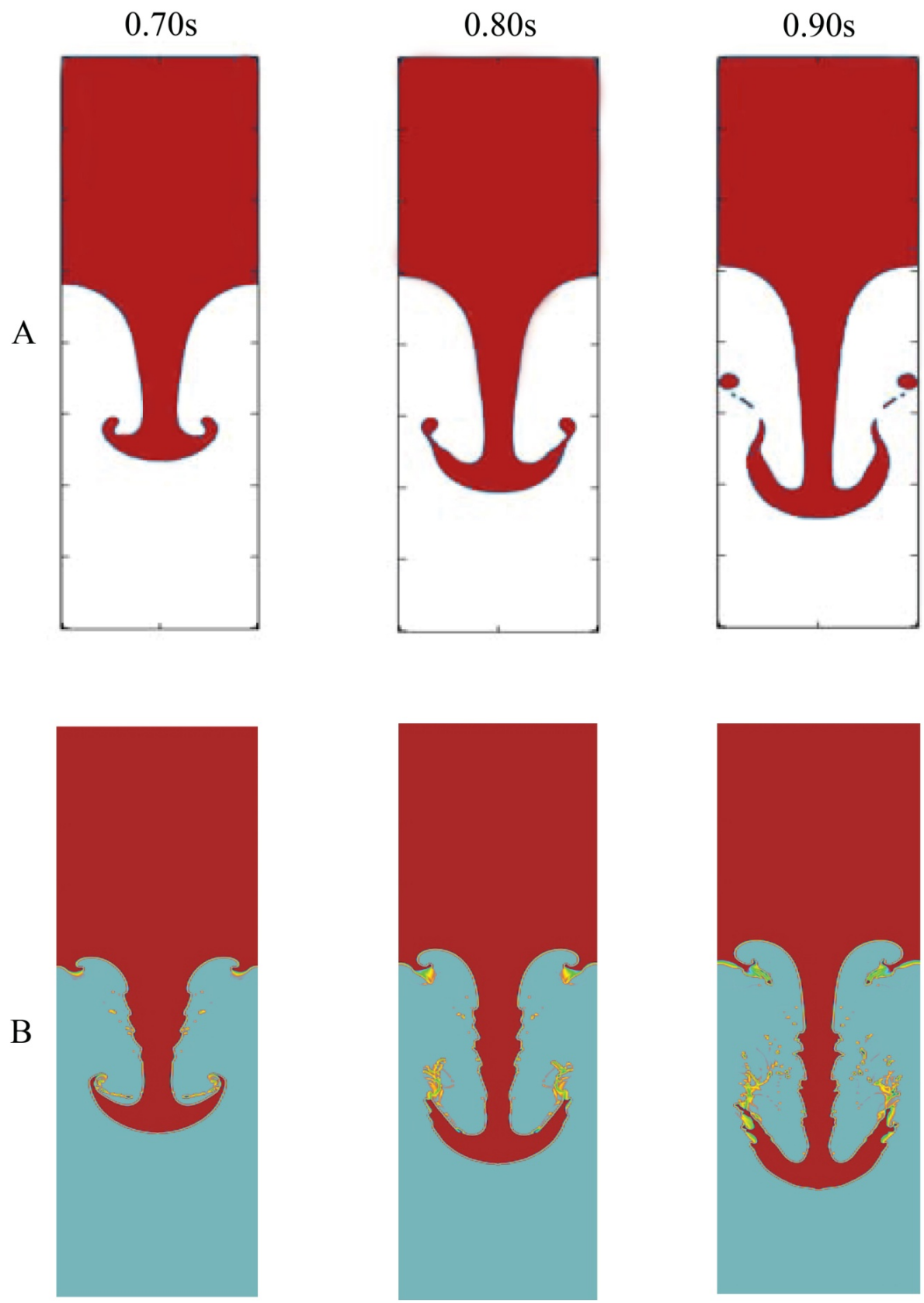

Figura 2 - Comparação dos campos de fração volumétrica. Em (A) os resultados obtidos por Jahanbakhsh et al. (2007), e em (B) os deste trabalho empregando o modelo tensorial. 


\section{QUEBRA DE BARREIRA}

\subsection{Setup}

Talvez um dos mais conhecidos problemas de testes em códigos multifásicos, a quebra de barreira consiste em uma coluna de fluido em repouso e subitamente ela colapsa (Chung, 2013; Hirt e Nichols, 1981; Shakibaeinia e Jin, 2011). A Figura 3 apresenta o domínio de cálculo com $a=0.05715 \mathrm{~m}$. Neste caso os fluidos são água ( $\rho=1000 \mathrm{~kg} / \mathrm{m}^{3} \mathrm{e}$ $\left.\mu=10^{-3} \mathrm{~Pa} \cdot \mathrm{s}\right)$, e ar $\left(\rho=1,00 \mathrm{~kg} / \mathrm{m}^{3}\right.$ e $\left.\mu=2 \cdot 10^{-5} \mathrm{~Pa} \cdot \mathrm{s}\right)$. Há um aumento significativo nas razões de densidade $(1000 \mathrm{x})$ e de viscosidade (50x). Isto implica em gradientes mais acentuados de velocidade, densidade e maiores taxas de deformação.

Neste caso, e malhas foram empregadas: Tensor e Tensor ST (41 x 161 nós), Tensor ST ref2x (81 x 321 nós) e Tensor ST ref4x (161 x 641 nós); onde ST implica na ativação do termo de tensão superficial (Brackbill, et al., 1992). O coeficiente de tensão superficial é considerado constate e igual a $0.071 \mathrm{~N} / \mathrm{m}$ com ângulo de contato prescrito igual a $90^{\circ}$. Pra a condição de abertura a pressão estática é fixada como sendo $0 \mathrm{~Pa}$ com fração volumétrica de ar igual a 1. O tempo físico é de $0,2 \mathrm{~s}$ com um passo de tempo de $0,0001 \mathrm{~s}$, com um tempo de computação de aproximadamente 10 dias.

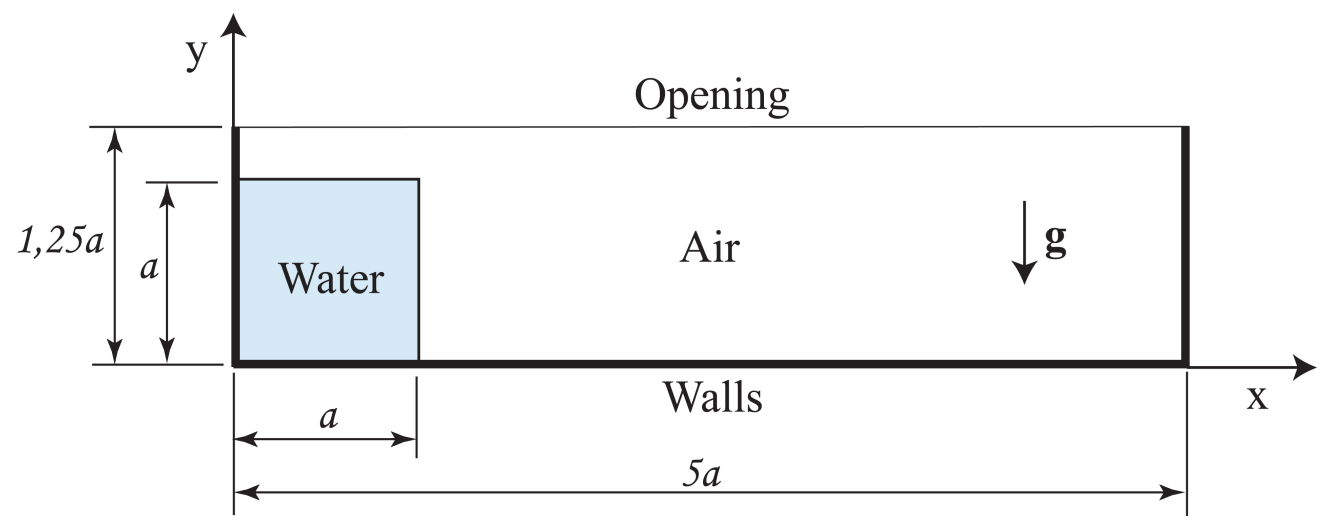

Figura 3 - Domínio de cálculo do rompimento de barreira com $a=0.05715 \mathrm{~m}$.

\subsection{Resultados e Discussão}

As evoluções temporais da altura da coluna de líquido e da frente de avanço são apresentadas na Figura 4 e comparadas com os resultados experimentais de Martin e Moyce (1952) e com os resultados numéricos de Jahanbakhsh, et al. (2007). O refino de malha tende a aproximar as curvas dos pontos experimentais, todavia, é sabido que os pontos experimentais possuem uma certa defasagem causada pelo diafragma que sustenta a coluna até o seu rompimento (Chung, 2013; Hirt e Nichols, 1981; Lind, et al., 2012; Park, et al., 2012).

O caso mais refinado (Tensor ST ref4x) foi o que teve o melhor desempenho, e o emprego da tensão superficial se mostrou mais eficiente quanto à estabilidade numérica. Os resultados em geral tiveram boa comparação com os dados experimentais reproduzindo bem 
o comportamento dinâmico do colapso da coluna de líquido.
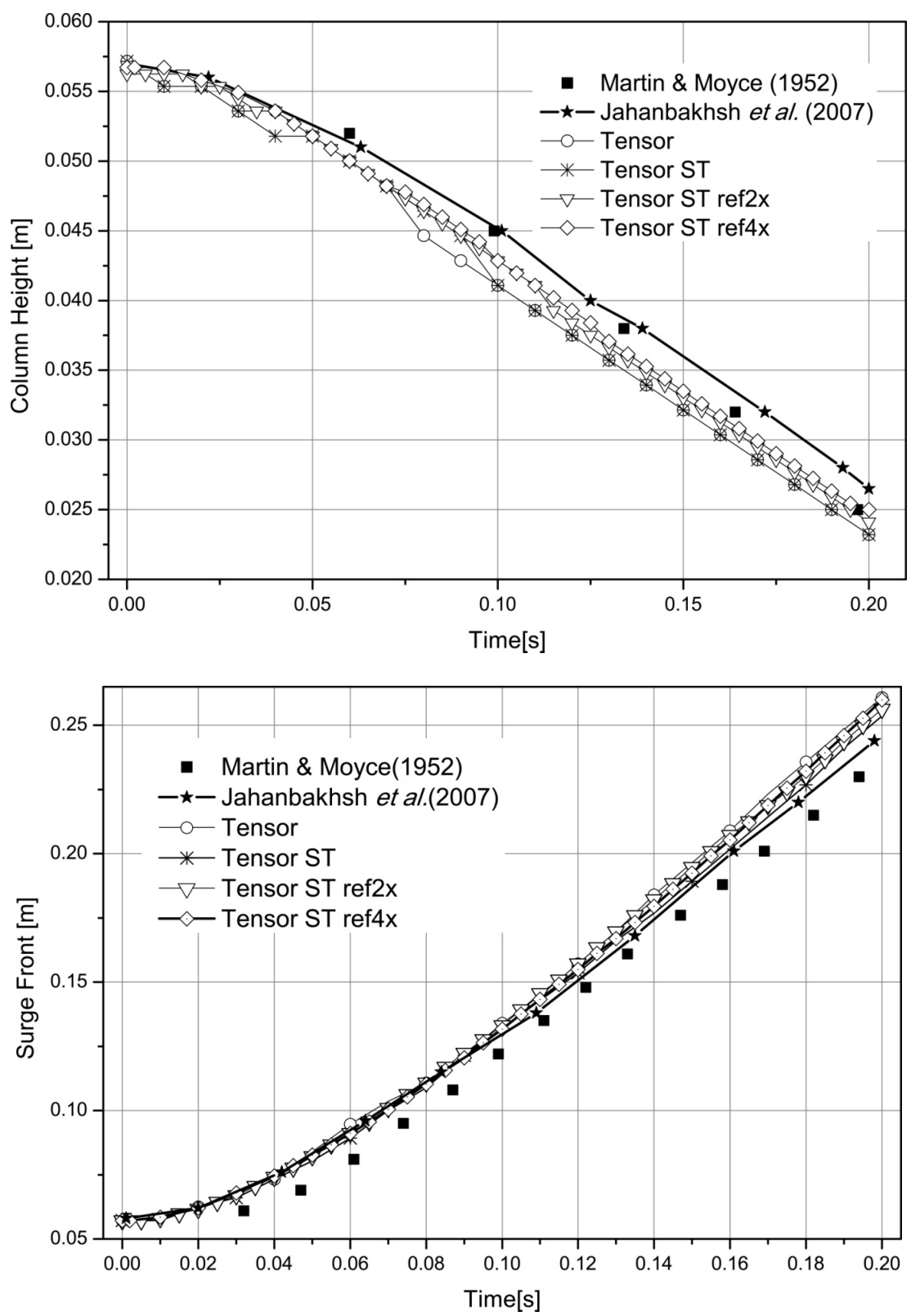

Figura 4 - Gráfico comparativo da evolução temporal da altura da coluna de líquido e da frente de avanço de líquido.

\section{CONCLUSÕES}

Uma abordagem para escoamentos estratificados foi proposta para o fechamento do termo de densidade de força interfacial no modelo de dois fluidos. 0 modelo emprega uma abordagem tensorial ara descrever a transferência de quantidade de movimento. 
Dois casos de teste escolhidos, instabilidade de Rayleigh-Taylor e rompimento de barreira, foram simulados e os resultados foram satisfatórios e com boa concordância com os dados disponíveis na literatura.

0 modelo tensorial descreve bem a dinâmica dos problemas escolhidos como mudança de morfologia estratificada e dispersa, e ainda permite que se calcule dinamicamente o coeficiente de arrasto e o empregue na formulação clássica se esta abordagem for preferida.

A viabilidade do modelo em outras situações deve ainda ser estudada para se determinar mais nitidamente as limitações e as vantagens do modelo.

\section{REFERÊNCIAS}

BARBOSA JR, J. R. Turbulência em sistemas bifásicos gás-líquido. Paper presented at the III Escola de Primavera de Transição e Turbulência, Florianópolis, 2002.

BRACKBILL, J. U., et al., A continuum method for modeling surface tension. Journal of Computational Physics, v. 100. (2), p. 335-354, 1992.

CHUNG, M.-H., An adaptive Cartesian cut-cell/level-set method to simulate incompressible two-phase flows with embedded moving solid boundaries. Computers \& Fluids, v. 71. (0), p. 469-486, 2013.

DEENDARLIANTO, et al., Application of a new drag coefficient model at CFD-simulations on free surface flows relevant for the nuclear reactor safety analysis. Annals of Nuclear Energy, v. 39. (1), p. 70-82, 2012.

DREW, D., et al., The analysis of virtual mass effects in two-phase flow. International Journal of Multiphase Flow, v. 5. (4), p. 233-242, 1979.

DREW, D. A., Mathematical flow modeling of two-phase flow. Annual Review of Fluid Mechanics, v. 15. p. 261-291, 1983.

. Effect of particle velocity flutuations on the inertia coupling in two-phase flow. Paper presented at the Constitutive Relationships and Models in Continuum Theories of Multiphase Flows, Huntsville, Alabama, 1989.

Analytical modeling of multiphase flows. In R. T. Lahey (Ed.), Boiling Heat Transfer: Modern Developments and Advances (pp. 31 - 83). Amsterdam; New York: Elsevier Science Publishers, 1992.

DREW, D. A., \& PASSMAN, S. L. Theory of multicomponent fluids. New York: Springer, 1999.

FOURNIER, E., et al., 2D pseudo-spectral parallel Navier-Stokes simulations of compressible Rayleigh-Taylor instability. Computers \& Fluids, v. 31. (4-7), p. 569587, 2002.

HÄNSCH, S., et al., A multi-field two-fluid concept for transitions between different scales of interfacial structures. International Journal of Multiphase Flow, v. 47. (0), p. 171-182, 2012.

HE, X., et al., A Lattice Boltzmann Scheme for Incompressible Multiphase Flow and Its Application in Simulation of Rayleigh-Taylor Instability. Journal of Computational Physics, v. 152. (2), p. 642-663, 1999. 
HIRT, C. W., \& NICHOLS, B. D., Volume of fluid (VOF) method for the dynamics of free boundaries. Journal of Computational Physics, v. 39. (1), p. 201-225, 1981.

HÖHNE, T. Experiments and Numerical Simulations of Horizontal Two-Phase Flow Regimes. Paper presented at the Seventh International Conference on CFD in the Minerals and Process Industries, Melbourn, Australia, 2009.

HÖHNE, T., \& VALLÉE, C. Numerical Prediction of Horizontal Stratified Flows. Paper presented at the 6th International Conference on CFD in Oil \& Gas, Metallurgical and Process Industries - SINTEF/NTNU, Thondhein, Norway, 2008.

Experiments and numerical simulations of horizontal two-phase flow regimes using an interfacial area density model. The Journal of Computational Multiphase Flows, v. 2. (3), p. 131-143, 2010.

JAHANBAKHSH, E., et al., Numerical simulation of three-dimensional interfacial flows. International Journal of Numerical Methods for Heat \& Fluid Flow, v. 17. (4), p. 384 - 404, 2007.

LIND, S. J., et al., Incompressible smoothed particle hydrodynamics for free-surface flows: A generalised diffusion-based algorithm for stability and validations for impulsive flows and propagating waves. Journal of Computational Physics, v. 231. (4), p. 1499-1523, 2012.

MARTIN, J. C., \& MOYCE, W. J., Part IV. An Experimental Study of the Collapse of Liquid Columns on a Rigid Horizontal Plane. Philosophical Transactions of the Royal Society of London. Series A, Mathematical and Physical Sciences, v. 244. (882), p. 312-324, 1952.

MEHRAVARAN, M., \& HANNANI, S. K., Simulation of incompressible two-phase flows with large density differences employing lattice Boltzmann and level set methods. Computer Methods in Applied Mechanics and Engineering, v. 198. (2), p. 223-233, 2008.

PARK, I., et al., Numerical investigation of the effects of turbulence intensity on dambreak flows. Ocean Engineering, v. 42. (0), p. 176-187, 2012.

REZENDE, R. V. P. Modelagem Matemática e Desenvolvimento de Metodologia Computacional para a simulação do Escoamento Bifásico de ar e Ferro-Gusa em Canal de Corrida de Alto-Forno. Dissertação (Mestrado). Engenharia Mecânica, Universidade federal de Santa Catarina, Florianópolis, 2008. xxx, 156 p.

- Modelo de Fechamento para o Tensor de Interface no Modelo de Dois Fluidos: Modelagem Matemática e Simulação. Tese (Doutorado). Engenharia Química, Universidade Federal de Santa Catarina, Florianópolis, 2014. 255 p.

REZENDE, R. V. P., et al. Modelo de Fechamento para Densidade de Força Interfcial do Modelo de Dois Fluidos sem Decomposição de Forças sobre a Interface. Paper presented at the COBEQ 2012, Buzios, Rj, 2012, Setembro, 2012.

SHAKIBAEINIA, A., \& JIN, Y.-C., A mesh-free particle model for simulation of mobile-bed dam break. Advances in Water Resources, v. 34. (6), p. 794-807, 2011. 